\title{
Fditorial
}

\section{The changing paradigm of dyslipidaemia management}

\author{
Ruvan A I Ekanayaka \\ National Hospital of Sri Lanka, Institute of Cardiology, Colombo, Sri Lanka
}

The 2013 American College of Cardiology (ACC)/American Heart Association (AHA) guideline on the treatment of blood cholesterol to reduce atherosclerotic cardiovascular risk in adults, ${ }^{(1)}$ effected a change in the paradigm of dyslipidaemia management, in that it no longer advocated a target based therapy but instead posited a primary clinical basis for initiation of statin therapy. The intensity of statin therapy too was guided by clinical risk criteria rather than by absolute values of the lipid profile.

Many clinicians were uncomfortable with the new paradigm. The 2011 European Society of Cardiology (ESC)/European Atherosclerosis Society (EAS) guidelines for the management of dyslipidaemia ${ }^{(2)}$ had given concrete lipid levels with reference to low density lipoprotein cholesterol (LDL- C),high density lipoprotein cholesterol(HDL-C), triglycerides (TG) and nonhigh density lipoprotein cholesterol(HDL-C) etc. which provided goals towards which clinicians could aim with clarity of purpose. This type of target value based paradigm has been the norm for the past 15-20 years.

Hence the unhappy response to the new paradigm of the 2013 ACC/AHA guideline which eliminated target based management. Clinicians as a group were familiar with managing metabolic diseases where laboratory testing gave an excellent guide regarding dosage, duration of therapyetc, which data would be accessible to both physician and patient. Diabetes mellitus is a good example of the management scenario where concrete laboratory values override clinical risk stratification as guide to therapy.
Taking the 2011 ESC/EAS guidelines for the management of dyslipidaemia as a representative specimen of the clinical practice guidelines of the pre-2013 era, we find that the LDL-C is selected as the primary target of therapy. The justification is based on the dose dependant reduction in ischaemic events seen with lowering of LDL-C. It is statistically determined that $40 \%$ reduction of LDL-C is associated with a $22 \%$ reduction of morbidity and mortality due to ischemic heart disease (IHD). ${ }^{(3)}$ Absolute values are also given. A LDLC level of less than $70 \mathrm{mg} \%$ or, a relative reduction of LDL-C 50\% from baseline, seem to offer the best benefit in cardiovascular event reduction. The specific target for non HDL-C is given as a $30 \mathrm{mg} \%$. This parameter includes most if not all the atherogenic particles (chylomicron remnants, verylow-density lipoprotein (VLDL), intermediate density lipoprotein (IDL), lipoprotein(a) $\left(\mathrm{LP}_{(\mathrm{a})}\right)$, and LDL. The $2011 \mathrm{ESC} /$ EAS guidelinedoes not set specific targets for HDL-C or triglycerides, as clinical trial evidence is lacking for determination of optimal values for these lipid constituents.

The 2011 ESC/EAS guideline further emphasises that the specific statin is of little consequence and that the type of statin must be selected based on it's ability to achieve the target LDL-C goal. Up titration of the statin dose is recommended until the LDL-C goal is achieved and this is made mandatory.

The 2013 ACC/AHA guideline did away with this mode of recommendations. The important determinant to effect the paradigm 
shift manifested in the 2013 ACC/AHA guideline, was the selection of evidence that was evaluated and utilized to prepare the guideline. Whereas the previous guidelines had taken into consideration the randomized clinical trials(RCT), epidemiological studies, basic science studies, clinical experience and genetics etc, the 2013 guideline utilized RCTs published since 1960 (in addition to systematic reviews and meta analyses of RCTs ). The expert panel studied 6 RCTs regarding primary prevention and 19 RCTs regarding secondary prevention.

The expert panel found good evidence that statin benefited 4 clinical groups:-

1. Individuals with clinical atherosclerotic cardiovascular disease (ASCVD)

2. Elevation of LDL-C $\geq 90 \mathrm{mg} \%$

3. 40-75 year old diabetics with LDL-C 70$189 \mathrm{mg} \%$

4. No ASCVD or diabetes mellitus (DM) but estimated 10year risk of ASCVD $\geq 7.5 \%$.

However the expert panel could not find evidence from the RCTs to recommended a specific LDL-C or non LDL-C target. Most, if not all,the RCTs had not titrated the statin therapy to achieve a target LDL-C level and the resultant effect was a dearth of RCT evidence for a specific LDL-C target.

In the absence of a goal for LDL-C, the clinician faces the problem of the intensity of statin therapy he needs to prescribe. The 2013 ACC/ AHA guideline classifies the intensity of statin therapy as high, moderate and lower. The expert panel used a calculated estimation of the percent LDL-C reduction for a specific statin - high intensity meant reducing LDL-L by $50 \%$, moderate intensity by $30 \%-50 \%$ and lower intensity by $<30 \%$.

Thus the 2013 ACC/AHA guideline changed the paradigm of dyslipidaemia management from a target laboratory value based model to a cardiovascular risk based one. The baseline LDL$\mathrm{C}$ level and the achieved LDL-C level were not deemed important in the crucial decisions regarding the initiation and up titration of statin therapy.
The latest updated guideline currently available is the $2016 \mathrm{ESC} / \mathrm{EAS}$ guideline for the management of dyslipidaemias. ${ }^{(4)}$ The compilers of this guideline have carefully considered the basis on which the 2013 ACC/AHA guidelines were constructed but have opted to retain the older paradigm of reducing LDL-C towards a target goal. Risk stratification takes an important place in the 2016 ESC/EAS guideline which has introduced a new risk category termed 'very high risk' group. Hence in this guideline, risk categorization comprises of four levels. The very high risk group would need a LDL-C level of $70 \mathrm{mg} \%$ or reduction of $50 \%$ from baseline LDL$\mathrm{C}$; for the high risk group LDL-C of $<100 \mathrm{mg} \%$ and for the low to moderate risk group an LDL-C of $<115 \mathrm{mg} \%$.

The 2016 Canadian Cardiovascular Society guideline for the management of dyslipidaemia for the prevention of cardiovascular disease in the adult ${ }^{(5)}$ closely follows the paradigm set in the 2016 ESC/EAS guideline but introduces abdominal aortic aneurysm $(>3 \mathrm{~cm}$ in diameter) into the indicated conditions.

The pleiotropic effects of statins are an important consideration in statin therapy irrespective of the baseline LDL-C levels, as the benefits of statin therapy exceed those predicted by cholesterol reduction alone. This well established fact is in favour of the $2013 \mathrm{ACC} /$ AHA paradigm for dyslipidaemia management. Clinicians must therefore take the pleiotropic effect benefits into account when planning the treatment of aggressive atherosclerotic disease. Although guidelines categorise risk on clinical grounds, atherosclerotic disease is dynamic and unstable by it's very nature and this factor has not been adequately addressed in the dyslipidaemia guidelines.

Another problem area neglected in the guidelines is the relevance of dense LDL particles ${ }^{(6)}$ and dysfunctional HDL. ${ }^{(7)}$ These are considered highly atherogenic but RCT evidence is lacking with regard to the targets and treatment modalities for either of these two lipid particles. Even the normal values for lipid parameters which are 
routinely estimated in the lipid profile appear to be fluid in that different values are given for different clinical scenarios.

It is therefore appropriate that the clinician use his clinical acumen to the maximum and not slavishly follow a given paradigm of dyslipidaemia management. He must be aware that both paradigms endorsed by the ESC/EAS and ACC/AHA have more similarities than differences and in fact could be complementary. It is the clinician who will know how the atherosclerotic disease has behaved in the past in a given patient and will be able to better predict how the disease will behave in the coming years (whatever the short comings of such a prediction might be). Based on this data and following any guideline, the best therapeutic option for each individual patient must be selected.

\section{REFERENCES}

1. Stone NJ, Robinson JG, Lichtenstein AH, et al. ACC/AHA Guideline on the Treatment of Blood Cholesterol to Reduce Atherosclerotic Cardiovascular Risk in Adults. A report of the
American College of Cardiology/American Heart Association Task Force on Practice Guidelines. Circulation 2014;129 (25 Suppl. 2):S1-45.

2. Reiner Z, Catapano AL, Graham I, et al. ESC/ EAS Guidelines for the management of dyslipidaemias. Eur Heart J 2011;32:1769-818.

3. Cholesterol Treatment Trialists' (CTT) Collaboration. Efficacy and safety of more intensive lowering of LDL cholesterol: a metaanalysis of data from 170000 participants in 26 randomised trials. Lancet 2010;376:1670-81.

4. Catapano AL, Graham I, De Backer G, et al. ESC/EAS Guidelines for the management of dyslipidaemias. Eur Heart J 2016; 37:2999-3058.

5. Anderson TJ, Gregoire J, Pearson GJ, et al. Canadian Cardiovascular Society Guidelines for the management of dyslipidemia for the prevention of cardiovascular disease in the adult.Canadian J Cardiol 2016;32:1263-82.

6. Wadhera RK, Steen DL, Khan I, et al. A review of low-density lipoprotein cholesterol, treatment strategies, and its impact on cardiovascular disease morbidity and mortality. J Clin Lipidol 2015;10:472-89. doi: 10.1016/j.jacl.2015.11. 010.

7. Serban C, Muntean D, Mikhailids DP, et al. Dysfunctional HDL: the journey from savior to slayer. Clin Lipid 2014;9:49-59. 